\title{
Prediction of Conducted Emissions in Satellite Power Buses
}

\author{
Giordano Spadacini, ${ }^{1}$ Flavia Grassi, ${ }^{1}$ Diego Bellan, \\ Sergio A. Pignari, ${ }^{1}$ and Filippo Marliani ${ }^{2}$ \\ ${ }^{1}$ Dipartimento di Elettronica, Informazione e Bioingegneria, Politecnico di Milano, 20133 Milano, Italy \\ ${ }^{2}$ European Space Research and Technology Centre (ESTEC), European Space Agency (ESA), 2201 AZ Noordwijk, Netherlands
}

Correspondence should be addressed to Sergio A. Pignari; sergio.pignari@polimi.it

Received 8 March 2015; Accepted 6 December 2015

Academic Editor: Joseph Majdalani

Copyright (C) 2015 Giordano Spadacini et al. This is an open access article distributed under the Creative Commons Attribution License, which permits unrestricted use, distribution, and reproduction in any medium, provided the original work is properly cited.

\begin{abstract}
This work reports a modeling methodology for the prediction of conducted emissions (CE) in a wide frequency range (up to $100 \mathrm{MHz}$ ), which are generated by $\mathrm{dc} / \mathrm{dc}$ converters and propagate along the power buses of satellites. In particular, the dc/dc converter seen as a source of CE is represented by a behavioral model, whose parameters can be identified by two unit-level experimental procedures performed in controlled test setups. A simplified multiconductor transmission-line (MTL) model is developed to account for the propagation of CE in shielded bundles of twisted-wire pairs used as power cables. The whole power system is represented by the interconnection of the circuit models of $\mathrm{dc} / \mathrm{dc}$ converters, cables, and Power Conditioning and Distribution Unit (PCDU). By solving the obtained network, frequency spectra of CE can be predicted. Experimental results are reported to substantiate the accuracy of the proposed unit-level dc/dc converter model and the MTL model of cables. Finally, a system-level test setup composed of three dc/dc converters connected to a PCDU is considered, and predicted CE are compared versus experimental measurements.
\end{abstract}

\section{Introduction}

Among several requirements for the verification of Electromagnetic Compatibility (EMC) in the Space sector, those concerning conducted emissions (CE) are aimed at enforcing strict limits to radiofrequency currents flowing in $\mathrm{dc}$ power buses in a wide frequency range extending up to $100 \mathrm{MHz}$ [1]. In order to explain how and where these disturbances are generated, Figure 1 reports a principle drawing of the typical topology of a satellite power system. The electric energy generated by the solar array feeds the Power Conditioning and Distribution Unit (PCDU), namely, an apparatus composed of several different modules (solar array power-point regulator, protection devices, battery regulators, power distribution for equipment/propulsion, etc.) devoted to electricpower management. From the PCDU, several power buses reach different functional units (e.g., a payload). These cables consist in bundles of twisted-wire pairs (TWPs) enclosed in a braided, metallic shield. The bus voltage is fixed (typically chosen between 28 and $50 \mathrm{~V}$ ). Finally, dc/dc converters inside each functional unit are used to obtain the desired voltage level (e.g., $5 \mathrm{~V}$ ) to feed specific circuit modules.

The switching activity of power-electronic valves inside $\mathrm{dc} / \mathrm{dc}$ converters (usually operating with switching frequencies higher than $100 \mathrm{kHz}$ ) implies the generation of harmonic disturbances in the frequency spectrum of currents. Hence, as shown in Figure 1, disturbance currents propagate along power cables back to the PCDU. These emissions potentially pose a threat to the correct operation of sensitive electronic equipment, due to crosstalk or other kinds of interference phenomena involving signal interconnects [2]. Additionally, long cables can turn the flowing CE into radiated electromagnetic fields in the closed, highly resonant interior of a satellite [2].

Specific testing activities are normally foreseen to assess $\mathrm{CE}$ of Space-qualified apparatus and equipment $[1,3,4]$. The conventional approach is usually based on two steps: (1) good engineering practices leading to the definition of unit-level requirements (i.e., specifications of test methods and quantitative $\mathrm{CE}$ limits for each unit to be integrated in 


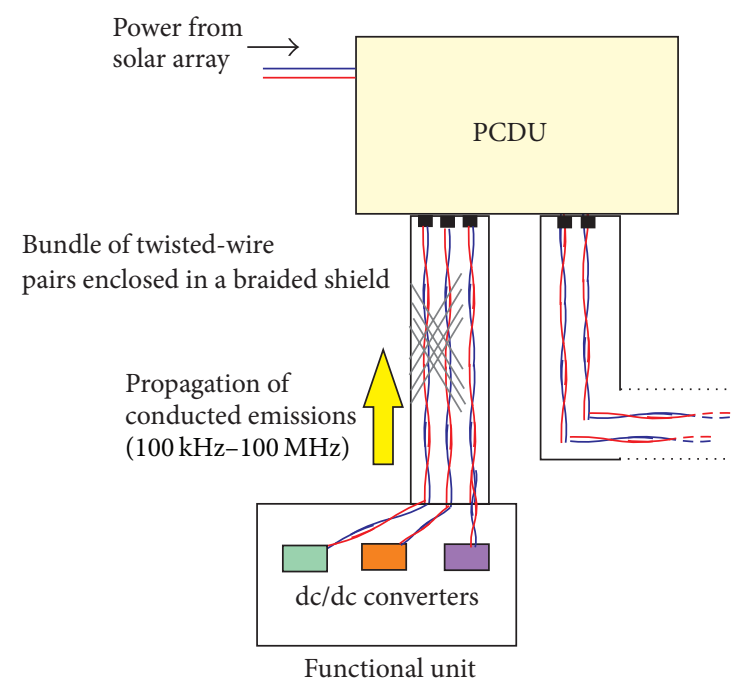

FIGURE 1: Structure of the dc power system in a satellite.

the system) and (2) extensive test campaigns carried out at unit and system level $[5,6]$.

Though such an experimental approach proves to be reliable and leads to excellent design achievements, nowadays prediction of CE through numerical simulation is an issue of increasing interest, since it could lead to reduction of costly test activities that could be replaced by simulation in a virtual-prototyping software environment. From a broader viewpoint, a simulation tool could be used either for preliminary EMC performance estimation during satellite mission-definition phase or for quantitative assessment of the impact of possible nonconformance detected during the manufacturing process. The first significant attempt in this direction was made in [7], where a behavioral model of $\mathrm{dc} / \mathrm{dc}$ converters used in spacecraft was derived by unitlevel measurements. This paper presents a new modeling methodology for the prediction of CE in spacecraft power systems which is more general than [7] since it involves an admittance-matrix representation of the $\mathrm{dc} / \mathrm{dc}$ converter passive model (instead of few impedances defined in [7]), and it accounts for the propagation of CE along the power buses. To this aim, two modeling problems are solved: (a) the representation of the $\mathrm{dc} / \mathrm{dc}$ converter as a source of CE and (b) the propagation of high-frequency currents along shielded TWP bundles, which are electrically long at the frequencies of interest (i.e., their physical length is comparable to or greater than the electromagnetic wavelength).

Concerning the representation of CE sources, different solutions for noise-source identification of power-electronic converters can be found in the literature [8-13]. In particular, advanced methodologies foresee the measurement of timedomain currents in unit-level setups, their transformation into the frequency domain via the Discrete Fourier Transform (DFT), and processing of results for the identification of frequency-domain circuit models [10-13]. This approach is exploited here for the development of a behavioral model of dc/dc-converter CE, presented in Section 2. Model parameters can be identified once and for all by simple experimental procedures at unit-level, namely, involving only the $\mathrm{dc} / \mathrm{dc}$ converter under analysis operating in a controlled test setup. The underlying idea is that a library of $\mathrm{dc} / \mathrm{dc}$ converter models can be readily created by using this methodology, due to the existence of a limited amount of Space-qualified suppliers and a relatively low number of different converter types.

For modeling unshielded TWP bundles, in [14] a simplified multiconductor transmission-line (MTL) model was based on the definition of equivalent per-unit-length (p.u.l.) parameters averaged over the twist pitch (i.e., the spatial period of twisting in TWPs). This approach is generalized in Section 3.1 to the case of shielded cables used in the satellite power system, by introducing additional simplifying assumptions and by validating the obtained results by comparison versus cable-characterization measurements.

In Section 3.2 the whole power system is represented by a network obtained by connecting dc/dc converters, cables, and PCDU models. The solution of the circuit is expressed in closed form and allows the prediction of frequency spectra of $\mathrm{CE}$ at system level. As a validating example, Section 4 reports a system-level test setup (composed of a PCDU and three $\mathrm{dc} / \mathrm{dc}$ converters and cables) for which predictions and measurements are compared and found in good agreement (the prediction error being less than $6 \mathrm{~dB}$ ). Finally, conclusions are drawn in Section 5.

\section{Unit-Level Model of the dc/dc Converter}

This section is aimed at developing a circuit model of the $\mathrm{dc} / \mathrm{dc}$ converter seen as a source of CE. Model parameters have to be inferred by suitable measurements performed at unit level, namely, in a controlled test setup involving only the operation of the $\mathrm{dc} / \mathrm{dc}$ converter under analysis and leaving any other component of the satellite power bus out of consideration. Moreover, measurements have to be performed at the external ports of the device seen as a blackbox object, so that there is no need for information about its internal structure (e.g., specific circuit topology and powerelectronic devices) which may be unavailable (e.g., in case of off-the-shelf components). As a practical result, such a test procedure can be applied once and for all for a number of $\mathrm{dc} / \mathrm{dc}$ converters of widest use, leading to the collection of a library of reusable $\mathrm{dc} / \mathrm{dc}$ converter models, which will be fundamental parts of the system-level circuit representation of the whole power bus described in the remainder of this paper.

2.1. Modeling Approach. From the viewpoint of circuit theory, the $\mathrm{dc} / \mathrm{dc}$ converter should be concerned as a time-varying nonlinear device. Nevertheless, at the frequencies of interest for CE (i.e., frequencies much greater than the fundamental switching frequency), a valid behavioral model can be represented by a linear multiport in the frequency domain [810]. In particular, active sources are used to represent highfrequency harmonics generated by valve switching, whereas an impedance network represents the passive part of the model, which is dominated by the effects of (a) linear parasitic components (e.g., inductances associated with wiring and capacitances between wires) and (b) the possible presence of 


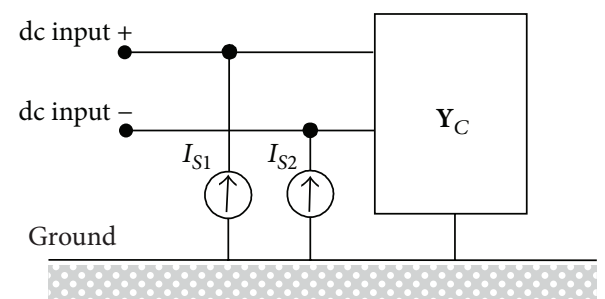

FIGURE 2: Behavioral model of the $\mathrm{dc} / \mathrm{dc}$ converter as a source of CE.

an integrated EMI filter (as often occurs for $\mathrm{dc} / \mathrm{dc}$ converters used in Space applications).

According to this approach, the $\mathrm{dc} / \mathrm{dc}$ converter seen as a source is represented here via the Norton-equivalent three-terminal network shown in Figure 2. Terminals are the plus/minus dc input pins and a ground reference representing the metallic case of the device (which must be taken into account since it behaves as an effective conductor for radiofrequency currents). CE are generated by equivalent current sources $I_{S 1}$ and $I_{S 2}$, whereas the passive part is modeled by a linear two-port network represented by the short-circuit admittance matrix $\mathbf{Y}_{C}$.

2.2. Identification of Model Parameters. For the identification of model parameters via experimental measurement, two test setups are proposed in Figure 3. In particular, the admittance matrix is directly measured at the input ports of the $\mathrm{dc} / \mathrm{dc}$ converter turned off (i.e., not fed by dc input power), as shown in Figure 3(a). A Vector Network Analyzer (VNA) is used to ensure accurate characterization at the high frequencies of interest (up to $100 \mathrm{MHz}$ ), which would not be achieved by using simpler measurement methods involving ordinary instrumentation unable to take into account effects of interconnections. Indeed, VNA ports are connected to converter terminals through coaxial cables, whose characteristics are automatically deembedded from measurements by the VNA calibration procedure. To this aim, a suitable interface structure (electrically short at the frequencies of interest) has been designed. The scattering-parameter ( $S$ parameter) matrix $\mathbf{S}_{C}$ of the structure is measured by the VNA, from which the admittance matrix is obtained as

$$
\mathbf{Y}_{\mathrm{C}}=R_{0}^{-1}\left(\mathbf{1}_{2}-\mathbf{S}_{\mathrm{C}}\right)\left(\mathbf{1}_{2}+\mathbf{S}_{\mathrm{C}}\right)^{-1},
$$

where $R_{0}=50 \Omega$ is the standard $S$-parameter reference resistance and $\mathbf{1}_{2}$ is the $2 \times 2$ unit matrix. This measurement method was found to be quite accurate, and interpretation of the obtained results for a number of $\mathrm{dc} / \mathrm{dc}$ converters showed an input admittance dominated by the frequency response of the integrated electromagnetic-interference (EMI) input filter (composed of inductors and capacitors). In this connection, one should note that the absence of dc biasing current in the proposed method could lead to inaccuracies, since magnetic cores of inductors may present a current-dependent magnetic permeability when working in their nonlinear region. Consequently, measured $S$-parameters may not reflect exactly the actual $S$-parameters in the operating condition of the $\mathrm{dc} / \mathrm{cd}$ converter. However, as filter inductors for EMI filters are properly designed to avoid saturation and nonlinear effects, the permeability variation should amount to a few percentages and can be practically neglected.

The second test setup shown in Figure 3(b) is subsequently used for the characterization of current sources $I_{S 1}$ and $I_{S 2}$. The dc/dc converter is fed by a linear power supply (i.e., not generating $\mathrm{CE}$ ); therefore it is turned on, and its dc output is connected to a suitable load (not shown in the figure) in order to set the power level of interest (usually, the nominal power). Indeed, the level of CE is strictly dependent on the operating point of the $\mathrm{dc} / \mathrm{dc}$ converter in terms of elaborated dc power. A Line-Impedance Stabilization Network (LISN) is inserted at the interface between the dc source and the $\mathrm{dc} / \mathrm{dc}$ converter. The aim of the LISN is to set a controlled and known impedance network seen by the $\mathrm{dc} / \mathrm{dc}$ converter at the frequencies of interest for $\mathrm{CE}$ analysis $(100 \mathrm{kHz}-100 \mathrm{MHz})$. In particular, let $\mathbf{Y}_{\mathrm{LISN}}$ be the admittance matrix forced by the LISN as shown in Figure 3(b). Suitable LISN configurations will be described in the next section. Here, it is worth mentioning that once an LISN is designed and physically constructed, its admittance matrix can be accurately characterized by a VNA using the same procedure outlined in Figure 3(a) for the dc/dc converter.

Finally, an oscilloscope equipped by broadband Halleffect current probes is used to acquire currents $I_{1}, I_{2}$ flowing out of converter terminals, which quantitatively represent CE according to Space standards [1]. These currents are acquired and stored in the time domain and subsequently converted into the frequency domain by using the DFT. As a result, one obtains the complex spectra of $I_{1}, I_{2}$ including not only information on current magnitudes, but also the relative phase shift. By solving the circuit model in Figure 3(b), the current sources in Figure 3(b) can be cast as

$$
\mathbf{I}_{S}=\left(\mathbf{1}_{2}+\mathbf{Y}_{C} \mathbf{Y}_{\text {LISN }}^{-1}\right) \mathbf{I},
$$

where $\mathbf{I}_{S}=\left[\begin{array}{ll}I_{S 1} & I_{S 2}\end{array}\right]^{T}$ and $\mathbf{I}=\left[\begin{array}{ll}I_{1} & I_{2}\end{array}\right]^{T}$ are column vectors of currents ( $T$ denotes transposition).

2.3. Experimental Validation of the Unit-Level Model. In order to exemplify the application of the proposed modeling approach in a practical case and to demonstrate its validity, some experimental results are here discussed. Figure 4 reports two possible LISN structures. Specifically, LISN A is similar to the capacitor network defined in the original version of MIL-STD-462 (methods CE01 and CE03, still in use in the Space sector) [15]. In principle, the use of capacitors allows $\mathrm{CE}$ to be enhanced (since capacitors have low impedance at high frequencies), so that accurate identification of current sources is expected by using the approach in Figure 3(b). Conversely, LISN B is similar to that specified in the ECSS Standard [1] and more representative of the typical lowfrequency impedance seen by $\mathrm{dc} / \mathrm{dc}$ converter input ports in typical satellite architecture (due to TWP and PCDU passive characteristics).

To demonstrate the effectiveness and validity of the proposed approach, a prototype of a full-bridge dc/dc converter with isolation transformer, resonant switching, and input EMI filter is characterized using the procedure explained in 


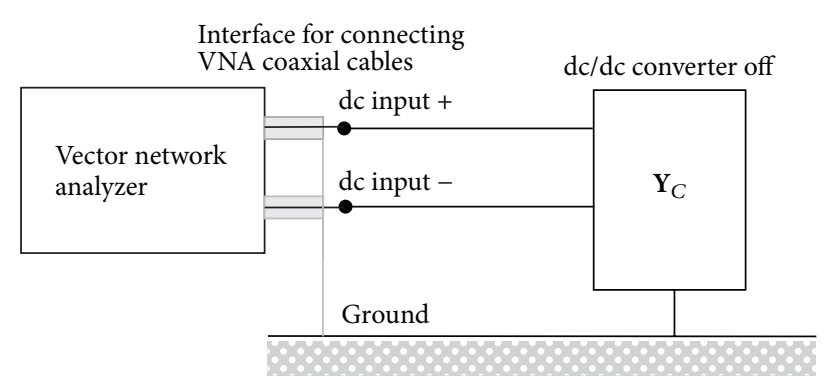

(a)

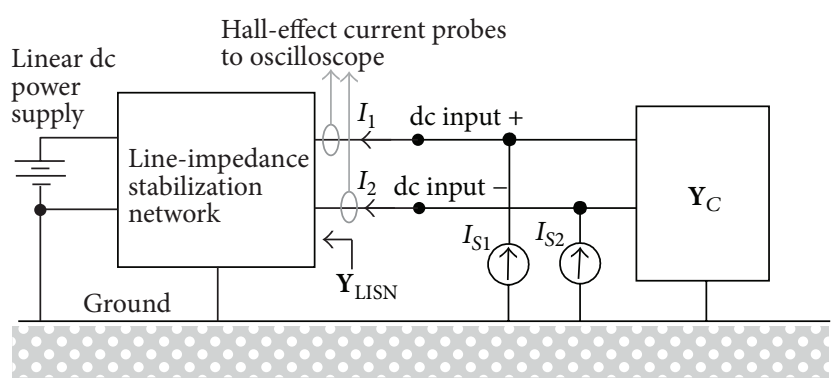

(b)

FIGURE 3: Unit-level test setups for the identification of parameters of the dc/dc converter behavioral model in Figure 2.

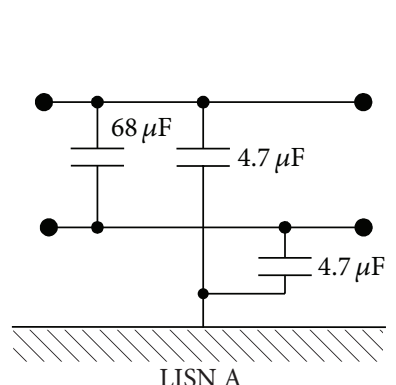

LISN A

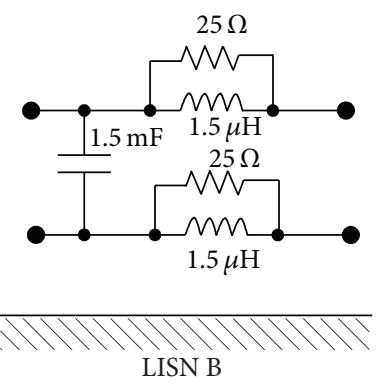

FIGURE 4: Two LISNs for the test setup in Figure 3(b).

previous section and involving LISN A. Subsequently, the circuit model in Figure 3(b) is used to predict CE currents $I_{1}, I_{2}$ when LISN B is inserted in the test setup (by inverting (2)), and results are compared versus measurements.

The magnitude of the four elements of the admittance matrix $\mathbf{Y}_{C}$ is plotted in Figure 5 versus frequency (a VNA Agilent E5071C was used for measurement). One can note that the equivalent network is reciprocal since $Y_{12}=Y_{21}$, according to the fact that the passive part of the $\mathrm{dc} / \mathrm{dc}$ converter model is dominated by the frequency response of the integrated EMI input filter composed of linear passive components. From Figure 5 one can also infer that the network is not perfectly symmetrical at the frequencies of interest, since $Y_{11} \neq Y_{22}$. Hence, the plus and minus dc terminals do not have perfectly equal short-circuit admittances to the metallic ground, most likely due to tolerances of component values as well as different parasitic elements (e.g., parasitic capacitances).

Figure 6 shows a picture of the test setup in Figure 3(b), which involved the use of LISN A in Figure 4 and a Tektronix DPO4104 oscilloscope equipped with Hall-effect current probes TCP0030. The magnitude of current sources $I_{S 1}, I_{S 2}$ resulting from (2) is plotted in Figure 7 . It is worth mentioning that the original spectra contained much more frequency points than those represented in Figure 7 (the frequency resolution of the DFT was $500 \mathrm{~Hz}$ ). Reduction of data points was intentionally done by means of an ad hoc computer code for the selection of emission peaks at harmonic frequencies. Indeed, since CE are ideally periodic signals in the time domain, their spectrum is composed of discrete harmonic components, that is, having frequency

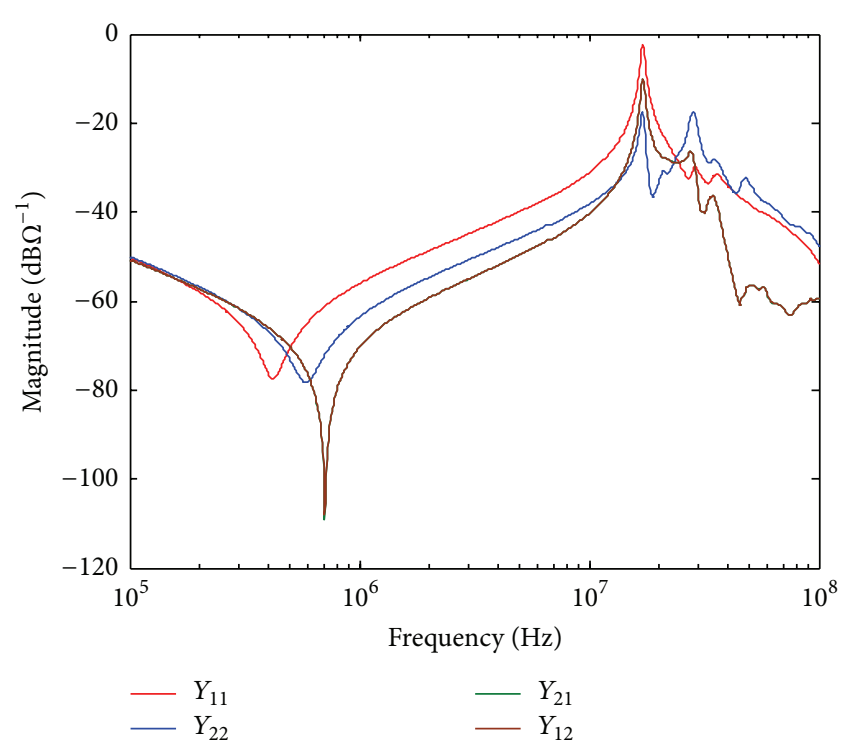

Figure 5: Magnitude of the four entries of the admittance matrix $\mathbf{Y}_{C}$ of the dc/dc converter versus frequency (note that $Y_{12}=Y_{21}$ ).

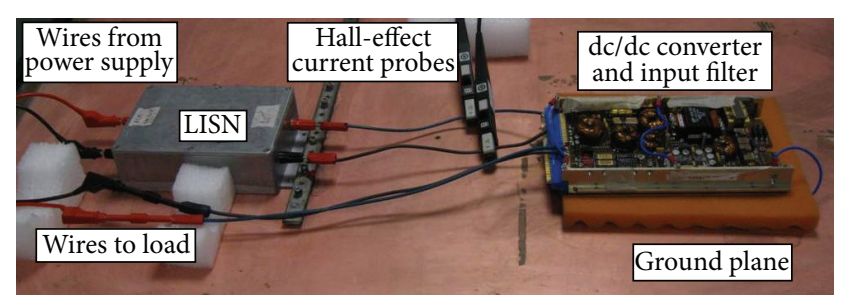

FIGURE 6: Picture of the unit-level test setup described in Figure 3(b).

which is multiple of the fundamental switching frequency of the $\mathrm{dc} / \mathrm{dc}$ converter (about $100 \mathrm{kHz}$ for the device under test). Therefore, only emission peaks are worth to be retained. In the spectrum representation of Figure 7 , the identified emission peaks are marked with dots, while straight lines are used to connect dots together. One can observe that the $\mathrm{dc} / \mathrm{dc}$ converter generates significant $\mathrm{CE}$ up to $30 \mathrm{MHz}$. Above this frequency there is mere broadband noise influenced by the sensitivity of the used instrumentation.

The data in Figures 5 and 7, together with phase angles of admittances $Y_{11}, Y_{22}, Y_{21}$, and $Y_{12}$, and of current sources 


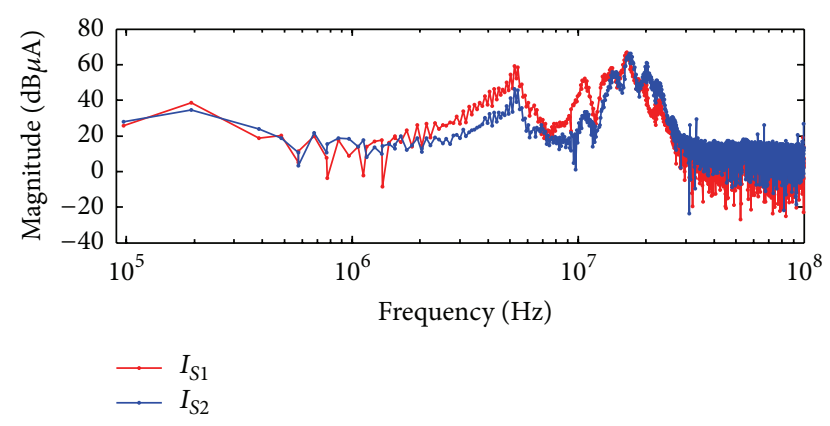

FIGURE 7: Magnitude of current sources $I_{S 1}$ and $I_{S 2}$ versus frequency (dots represent harmonics components).
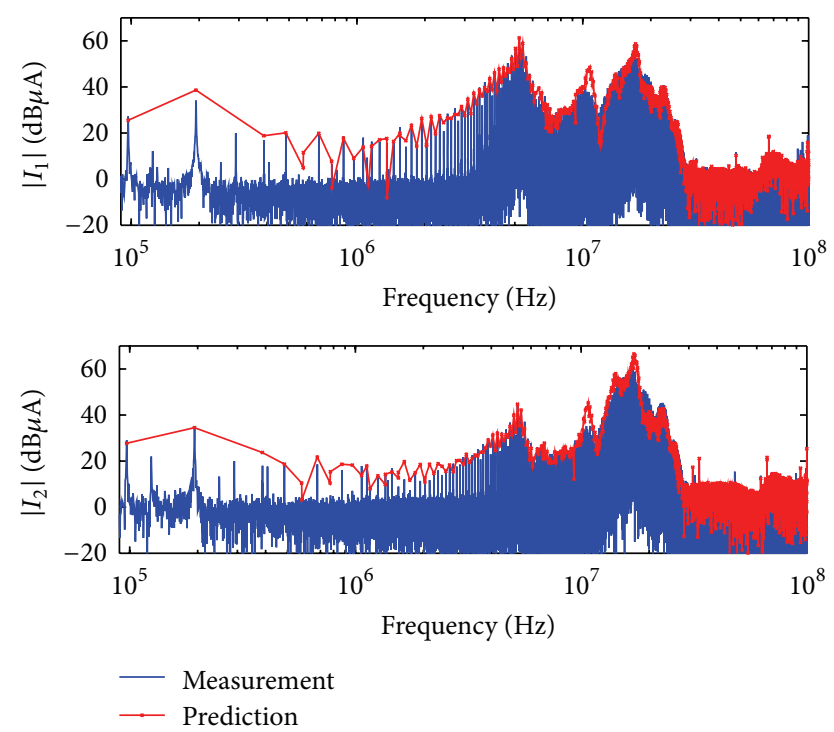

Figure 8: Magnitude of CE $I_{1}$ and $I_{2}$ in the test setup defined in Figure 3(b) and involving LISN B in Figure 4. Blue lines refer to experimental measurements (i.e., DFT of currents acquired by the oscilloscope). Red dots and lines refer to predictions obtained by solving the circuit model with the identified parameters of the $\mathrm{dc} / \mathrm{dc}$ converter.

$I_{S 1}, I_{S 2}$ (not plotted here for the sake of brevity) are stored in computer files and form a complete set of identified frequency-dependent parameters for the $\mathrm{dc} / \mathrm{dc}$ converter model in Figure 2.

To demonstrate model validity, measurement of $\mathrm{CE}$ was repeated in the test setup of Figure 3(b) involving LISN $B$ (instead of LISN A used for the identification of model parameters). The obtained currents spectra $I_{1}$ and $I_{2}$ are plotted in Figure 8 and compared versus the prediction obtained by solving the circuit model, that is, by computing

$$
\mathbf{I}=\left(\mathbf{1}_{2}+\mathbf{Y}_{C} \mathbf{Y}_{\text {LISN }}^{-1}\right)^{-1} \mathbf{I}_{S}
$$

Experimental validations demonstrate good agreement of predictions and measurements, even considering nonideal phenomena affecting model accuracy, including measurement uncertainty related to instrumentation and to test setups. The average error is mostly lower than $6 \mathrm{~dB}$, which is adequate for practical aims of designers involved in EMC analyses (e.g., estimation of interference levels and definition of EMC requirements for subsystems).

\section{System-Level Model of the Power Bus}

Shielded bundles of TWPs interconnecting the PCDU and $\mathrm{dc} / \mathrm{dc}$ converters are fundamental parts of the satellite power system. While their impact is merely limited to resistive losses and inductive behavior from a functional standpoint (e.g., in dc), their complex frequency response has great importance in EMC, since it influences generation and propagation of CE. Specifically, at the frequencies of interest for CE, the physical length of cables is comparable to or greater than the electromagnetic wavelength. Therefore cables have to be modeled as distributed-parameter circuits through MTL theory [16]. This section presents an MTL model for typical TWP cables used in satellite power buses and assesses its range of validity via experimental measurements. Finally, by combining models of $\mathrm{dc} / \mathrm{dc}$ converters and cables, a systemlevel model of the power bus is obtained.

3.1. Modeling of Shielded TWP Cables. A typical cable composed of TWPs enclosed in a braided shield and a protective sheath is shown in Figure 9(a). The cross section is sketched in Figure 9(b) for a number of three TWPs (for mere exemplification without loss of generality). It is important noting that the knowledge about geometrical and electrical properties of such cable structures (often hand made on the basis of custom requirements) is always affected by large uncertainty. Indeed, the geometry of power cables is not accurately controlled as for telecommunication cables, which are designed to achieve good propagation properties at high frequencies (e.g., the desired characteristic impedance). Particularly, since the shield is braided, its shape is not perfectly circular, and it is subject to changes along the cable length. Moreover, TWPs are bundled together using no dielectric fillers or any other mean to stabilize their position in the cross section; hence TWPs may follow random paths along the bundle length. For this reason, and due to the helical twisting of wires in each TWP, the MTL is nonuniform [16]. Finally, the dielectric medium is nonhomogeneous, due to the simultaneous presence of air space and dielectric coating of wires [16]. As a result, modeling of such cable structures at radio frequencies is a challenging trade-off between complexity, representativeness, and accuracy.

The proposed model is based on a number of simplifying assumptions aimed at retaining only dominant phenomena involved in the propagation of $\mathrm{CE}$, with a minimum number of involved geometrical/electrical parameters. First of all, the simplified homogeneous cross section shown in Figure 9(c) is considered, where an effective dielectric medium (with permittivity $\varepsilon$ greater than that of air) is used to fill the space inside a solid circular shield (sized so to enclose all TWPs, which are symmetrically arranged). Geometrical parameters are (a) the wire radius $r_{w}$, (b) the wire separation $s$ in each pair, and (c) the separation $s_{b}$ between TWPs in the bundle. All media are lossless (this assumption simplifies modeling 


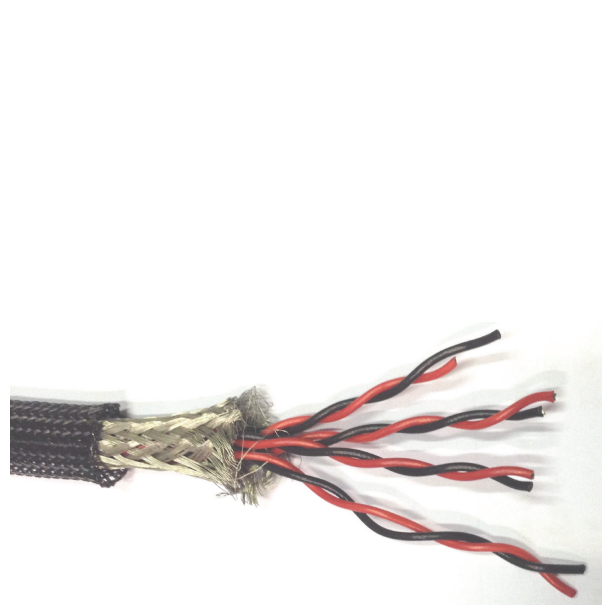

(a)

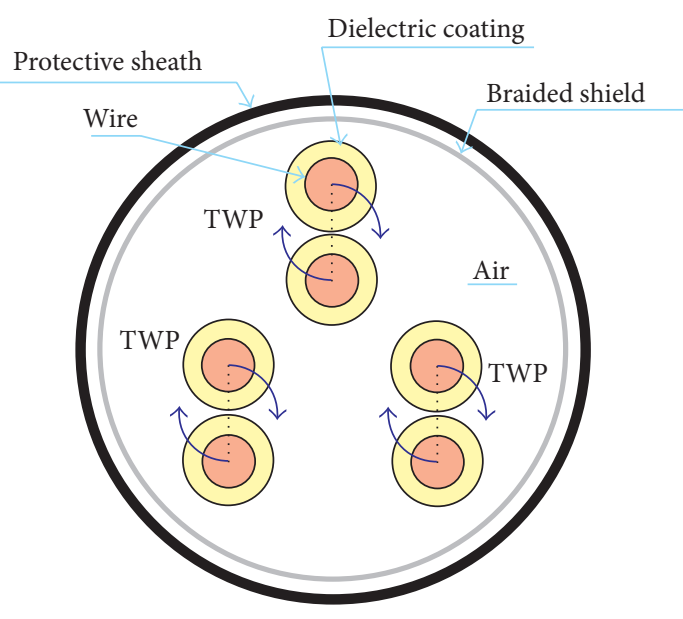

(b)

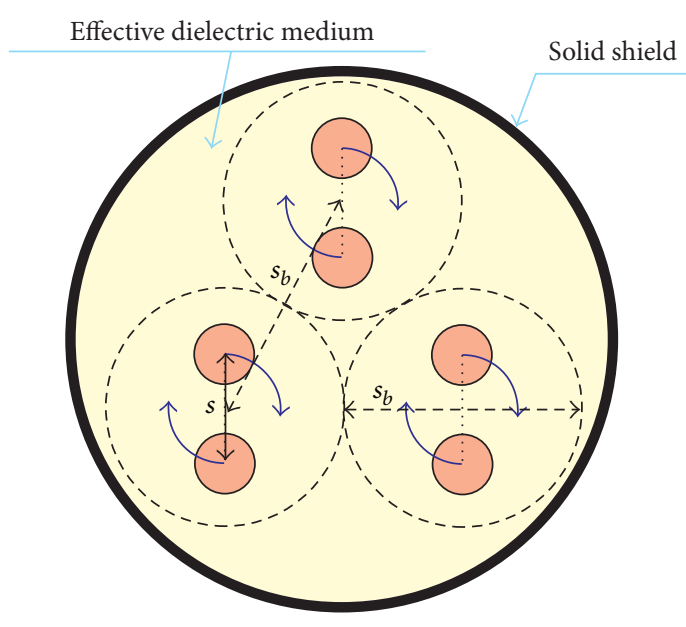

(c)

FIGURE 9: Shielded TWP cable. (a) Picture; (b) actual cross section; and (c) simplified cross section for modeling purposes.

efforts while leading to precautionary overestimation of $\mathrm{CE}$ ). Finally, in order to model the nonuniform line via MTL equations, the modeling methodology developed in [14] for TWP bundles is applied, which is based on the definition of averaged p.u.l. parameters to obtain an equivalent uniform MTL. Namely, say $N$ is the number of TWPs, the $2 N \times$ $2 N$ p.u.l. inductance matrix $\mathbf{L}$ is partitioned into $2 \times 2$ submatrices as

$$
\mathbf{L}=\left[\begin{array}{cccc}
\overline{\mathbf{L}}_{1} & \overline{\mathbf{M}}_{12} & \cdots & \overline{\mathbf{M}}_{1 N} \\
\overline{\mathbf{M}}_{12} & \overline{\mathbf{L}}_{2} & \cdots & \overline{\mathbf{M}}_{2 N} \\
\vdots & \vdots & \vdots & \vdots \\
\overline{\mathbf{M}}_{1 N} & \overline{\mathbf{M}}_{2 N} & \cdots & \overline{\mathbf{L}}_{N}
\end{array}\right]
$$

Each submatrix $\overline{\mathbf{L}}_{n}, n=1,2, \ldots, N$, is a $2 \times 2$ symmetric matrix with two equal in-diagonal, $\bar{l}_{n}$, and off-diagonal, $\bar{l}_{M, n}$, entries that represent the averaged p.u.l. self- and mutual inductances of the wires belonging to the $n$th pair, respectively. Additionally, each submatrix $\overline{\mathbf{M}}_{n k}, n, k=1,2, \ldots, N$, is a $2 \times 2$ matrix containing four equal entries $\bar{l}_{n k}$, representing averaged p.u.l. mutual inductances between wires belonging to the $n$th and $k$ th TWP. The line placed above these symbols highlights that quantities represent averages over a twist period; that is,

$$
\begin{aligned}
\bar{l}_{n} & =\frac{1}{2 \pi} \int_{0}^{2 \pi} l_{n}\left(\varphi_{n}\right) d \varphi_{n}, \\
\bar{l}_{M, n} & =\frac{1}{2 \pi} \int_{0}^{2 \pi} l_{M n}\left(\varphi_{n}\right) d \varphi_{n}, \\
\bar{l}_{n k} & =\frac{1}{4 \pi^{2}} \iint_{0}^{2 \pi} l_{n k}\left(\varphi_{n}, \varphi_{k}\right) d \varphi_{n} d \varphi_{k},
\end{aligned}
$$

where $l_{n}\left(\varphi_{n}\right), l_{M n}\left(\varphi_{n}\right)$, and $l_{n k}\left(\varphi_{n}, \varphi_{k}\right)$ are variable p.u.l. inductances depending on the tilt angle $\varphi_{n}$ of the $n$th TWP, which describes the rotation (simply represented with blue arrows in Figure 9) of the pair. Integrals (5) can be conveniently computed through numerical methods [14].

As a final result, the cable is modeled by the multiport network in Figure 10, where the voltage vector $\mathrm{V}(\mathscr{L})$ and current vector $\mathbf{I}(\mathscr{L})$ at the end terminals (where $\mathscr{L}$ denotes the cable length), composed of $2 \mathrm{~N}$ entries, are related to those 


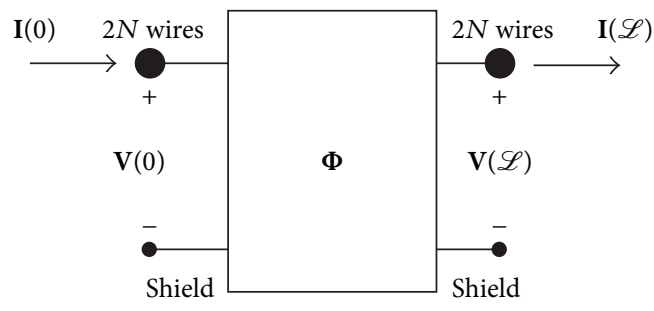

FIGURE 10: Multiport network modeling the cable composed of $N$ TWPs enclosed in a shield. The thick line is representative for $2 \mathrm{~N}$ wires.

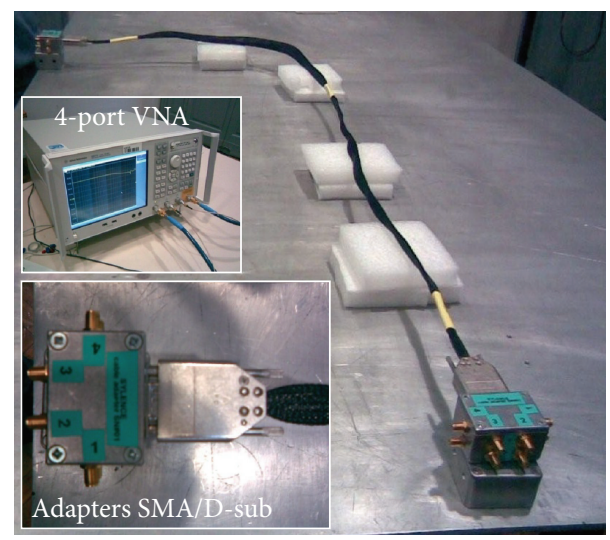

FIGURE 11: Experimental setup for the characterization of shielded TWP cables used in satellite power buses.

of the starting terminals $\mathbf{V}(0)$ and $\mathbf{I}(0)$, respectively, through the chain parameter matrix $\Phi$ having size $4 N \times 4 N$ in the form

$$
\begin{aligned}
{\left[\begin{array}{c}
\mathbf{V}(\mathscr{L}) \\
\mathbf{I}(\mathscr{L})
\end{array}\right] } & =\boldsymbol{\Phi} \cdot\left[\begin{array}{l}
\mathbf{V}(0) \\
\mathbf{I}(0)
\end{array}\right], \\
\boldsymbol{\Phi} & =\left[\begin{array}{ll}
\boldsymbol{\Phi}_{11} & \boldsymbol{\Phi}_{12} \\
\boldsymbol{\Phi}_{21} & \boldsymbol{\Phi}_{22}
\end{array}\right] \\
& =\left[\begin{array}{cc}
\cos (\beta \mathscr{L}) \mathbf{1}_{2 N} & -j \mathbf{Z}_{C} \sin (\beta \mathscr{L}) \\
-j \mathbf{Z}_{C}^{-1} \sin (\beta \mathscr{L}) & \cos (\beta \mathscr{L}) \mathbf{1}_{2 N}
\end{array}\right],
\end{aligned}
$$

where $j$ is the imaginary unit, $\beta=2 \pi f / v$ is the propagation constant ( $f$ is the frequency, $v=\left(\varepsilon \mu_{0}\right)^{-1}$ is the propagation speed, $\varepsilon$ is the effective dielectric permittivity, and $\mu_{0}$ is the magnetic permeability of vacuum), $\mathbf{1}_{2 N}$ denotes the $2 \mathrm{~N} \times 2 \mathrm{~N}$ unit matrix, and $\mathbf{Z}_{C}=v \mathbf{L}$ is the characteristic impedance matrix.

The proposed model was validated versus measurement carried out within an extensive experimental campaign aimed at the characterization of shielded cables used in satellite power buses (a picture of the experimental setup is reported in Figure 11). During this activity, a four-port VNA was used to acquire the scattering parameters of several samples of cables. To this end, suitable adapters were designed to interface SMA coaxial connectors of the VNA to the Dsub connectors used in satellite power buses (see Figure 11). The cables under test were composed of $N=4$ TWPs,
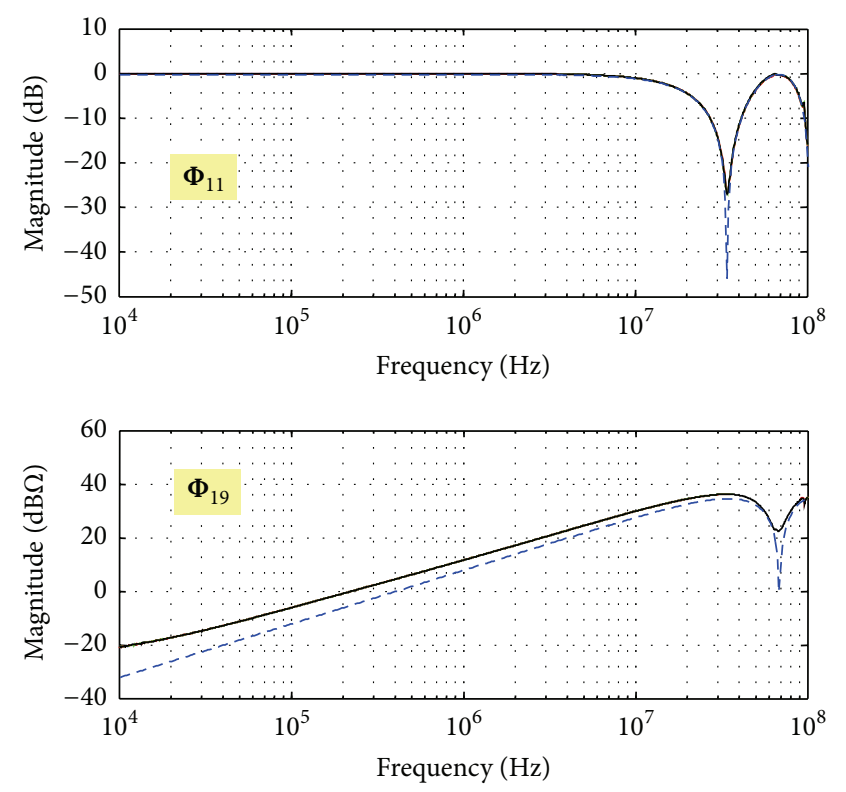

FIGURE 12: Comparison of model results (dashed blue line) and measurements (solid lines) for two entries of the chain-parameter matrix.

with axial length $1.5 \mathrm{~m}$ and minimum number of 19 twists per meter. Cables were placed at constant height $(5 \mathrm{~cm})$ above a metallic ground plane (in contact with the cable shield through the metallic case of D-sub connectors and the adapter). Due to helical twisting of wires, the harness actual length to be considered in (7) was greater than the axial length; namely, $\mathscr{L}=1.8 \mathrm{~m}$. The wire radius was $r_{w}=$ $0.375 \mathrm{~mm}$, and the separation $s=2.2 r_{w}$. The separation of TWPs was set to the minimum value $s_{b}=2 s$ (for geometrical consistency). The only parameter arbitrarily selected so to optimize the agreement of the model with measurements was the unknown effective electric permittivity, for which a value of $\varepsilon=1.5 \varepsilon_{0}$ was found satisfactory (it represents an intermediate value between the permittivity $\varepsilon_{0}$ of vacuum and the permittivity $3.5 \varepsilon_{0}$ of the actual wire coating).

The $S$-parameter matrix acquired via VNA measurement was transformed into the chain-parameter matrix, whose entries were compared versus the outcome (7) of the proposed modeling approach. Note that the large number of complex-valued frequency-dependent matrix entries (i.e., $16 N^{2}$ ) prevents reporting here a complete set of results. However, for the sake of exemplification, Figure 12 shows two plots with predictions and measurements for the magnitude of selected entries of (7), namely, $\Phi_{11}$ (i.e., first row and column of submatrix $\boldsymbol{\Phi}_{11}$ ) and $\Phi_{19}$ (i.e., first row and column of submatrix $\Phi_{12}$ ). In particular, $\Phi_{11}$ exhibits excellent agreement in the whole frequency range (except for the sharp notch at about $35 \mathrm{MHz}$ ). Since notches and resonances of the frequency response represent very narrow frequency ranges characterized by high sensitivity, possible model inaccuracy is unavoidable. Conversely, $\Phi_{19}$ shows a discrepancy of the lowfrequency slope predicted by the model (i.e., $+20 \mathrm{~dB} /$ decade) with respect to the lower slope of measurements. One can 


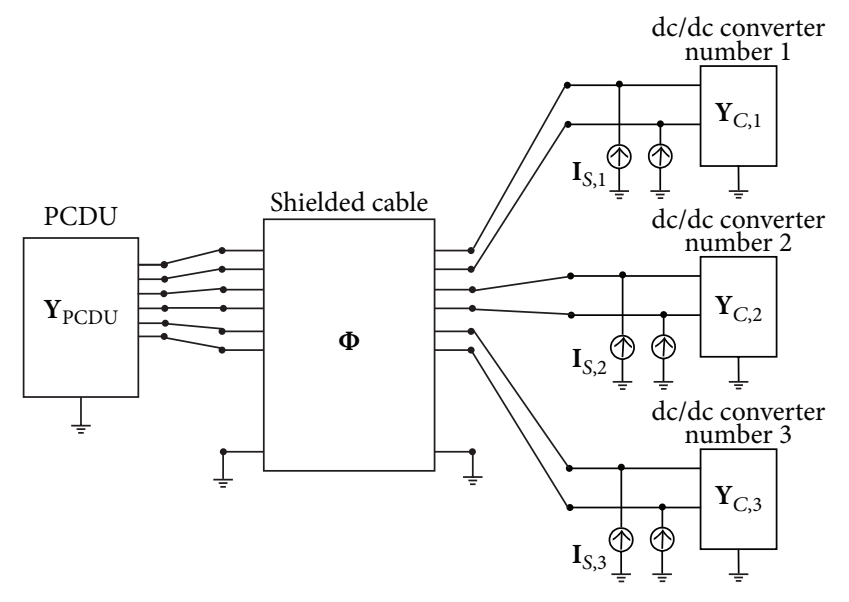

FIGURE 13: Power-bus network, involving multiport circuits representative of the PCDU, the shielded cable, and the dc/dc converters.

show that this discrepancy originates from neglecting resistive losses in the braided shield. However, as far as frequencies greater than $100 \mathrm{kHz}$ are concerned, the error is less than $6 \mathrm{~dB}$, which is in line with practical objectives of the proposed analysis. In general, for all entries of (7), discrepancies do not exceed $6 \mathrm{~dB}$ on average.

3.2. Solution of the Power-Bus Network. Once unit-level models of $\mathrm{dc} / \mathrm{dc}$ converters and cables are available, the solution of the power-bus network is a simple matter. Figure 13 shows the obtained system-level circuit model, resulting from the interconnection of dc/dc converters, a shielded TWP bundle, and the PCDU. Without loss of generality, the example refers to three $\mathrm{dc} / \mathrm{dc}$ converters fed by a common cable, representing a branch (see Figure 1) of the power system (the presence of multiple and separate shielded cables is a straightforward extension).

The PCDU is modeled as a passive network characterized by an admittance matrix $\mathbf{Y}_{\mathrm{PCDU}}$, which can be experimentally characterized via VNA measurements as explained in Section 2 for matrix $\mathbf{Y}_{C}$ of the dc/dc converter. The target of the proposed analysis is the computation of $\mathrm{CE}$, that is, currents $\mathbf{I}(0)$ and $\mathbf{I}(\mathscr{L})$ flowing at cable terminals (see Figure 10 ). To this aim, let us define the equivalent admittance-matrix and current source vectors for $\mathrm{N} \mathrm{dc/dc} \mathrm{converters} \mathrm{as}$

$$
\begin{gathered}
\mathbf{Y}_{C}=\left[\begin{array}{cccc}
\mathbf{Y}_{\mathbf{C}, 1} & \mathbf{0}_{2} & \cdots & \mathbf{0}_{2} \\
\mathbf{0}_{2} & \mathbf{Y}_{\mathbf{C}, 2} & \cdots & \mathbf{0}_{2} \\
\vdots & \vdots & \vdots & \vdots \\
\mathbf{0}_{2} & \mathbf{0}_{2} & \cdots & \mathbf{Y}_{\mathbf{C}, N}
\end{array}\right] \\
\mathbf{I}_{S}=\left[\begin{array}{llll}
\mathbf{I}_{\mathbf{S}, 1} & \mathbf{I}_{\mathbf{S}, 2} & \cdots & \mathbf{I}_{\mathbf{S}, N}
\end{array}\right]^{T},
\end{gathered}
$$

where $\mathbf{0}_{2}$ is the $2 \times 2$ null matrix. By solving the circuit model in Figure 13, the following sets of equations are obtained:

$$
\begin{aligned}
& {\left[\boldsymbol{\Phi}_{21}-\boldsymbol{\Phi}_{22} \mathbf{Y}_{\mathrm{PCDU}}-\mathbf{Y}_{\mathrm{C}} \boldsymbol{\Phi}_{11}+\mathbf{Y}_{\mathrm{C}} \boldsymbol{\Phi}_{12} \mathbf{Y}_{\mathrm{PCDU}}\right] \cdot \mathbf{V}(0)} \\
& \quad=-\mathbf{I}_{\mathbf{S}}
\end{aligned}
$$

$$
\begin{aligned}
& \mathbf{V}(\mathscr{L})=\left[\boldsymbol{\Phi}_{11}-\boldsymbol{\Phi}_{12} \mathbf{Y}_{\mathrm{PCDU}}\right] \cdot \mathbf{V}(0), \\
& \mathbf{I}(0)=-\mathbf{Y}_{\mathrm{PCDU}} \cdot \mathbf{V}(0), \\
& \mathbf{I}(\mathscr{L})=-\mathbf{I}_{\mathbf{S}}+\mathbf{Y}_{\mathrm{C}} \cdot \mathbf{V}(\mathscr{L}) .
\end{aligned}
$$

Matrix expression (9) represents a linear system of $2 \mathrm{~N}$ algebraic equations, which can be solved for the $2 \mathrm{~N}$ terminal voltages in vector $\mathbf{V}(0)$. Once these equations are solved, vector $\mathbf{V}(\mathscr{L})$ is obtained from $(10)$, and $\mathrm{CE} \mathbf{I}(0)$ and $\mathbf{I}(\mathscr{L})$ are computed from terminal relationships (11) and (12), respectively.

\section{Experimental Validation}

This section reports experimental measurements aimed at assessing the performance of the developed modeling tool in prediction of $\mathrm{CE}$ at system level. An ad hoc device representing the "functional unit" in Figure 1 was designed and constructed. It was composed of three off-the-shelf $\mathrm{dc} / \mathrm{dc}$ converters with different topologies mounted on a heat sink and suitable metallic strips holding both coaxial (SMA) and $\mathrm{D}$-sub connectors to allow easy interconnection of $\mathrm{dc} / \mathrm{dc}$ converters with the VNA and power cables. Dc/dc converters were fed by a $28 \mathrm{~V}$ input, and the output voltages were $12 \mathrm{~V}$, $28 \mathrm{~V}$, and $48 \mathrm{~V}$, respectively. Each $\mathrm{dc} / \mathrm{dc}$ converter provided a maximum output power of $250 \mathrm{~W}$, set and controlled by an electronic load. Model parameters of the involved $\mathrm{dc} / \mathrm{dc}$ converters were identified according to the procedure described in Section 2. In particular, LISN A in Figure 4 was used in the test setup of Figure 3(b). A picture of the unitlevel test setup (for measurement of the input admittance) is reported in Figure 14(a).

Subsequently, the $\mathrm{dc} / \mathrm{dc}$ converters were put in the system-level test setup shown in Figure 14(b), where they could operate simultaneously, being fed by a PCDU embedded in the Electrical Power System (EPS) of a small satellite. Three shielded TWP cables were used for interconnecting the EPS to each dc/dc converter. Radiofrequency currents at the end of these cables ( $\mathrm{dc} / \mathrm{dc}$ converter side) were measured by an oscilloscope equipped with Hall-effect current probes and transformed into the frequency domain via the DFT. An example of the obtained results is shown in Figure 15, where measurement and prediction of noise currents flowing in the plus dc terminal of $\mathrm{dc} / \mathrm{dc}$ converter $\# 1$ are compared. Here, two useful features were added to the modeling approach: (a) the possibility of setting an arbitrary minimum frequency of interest $(300 \mathrm{kHz}$ in Figure 15) during model-identification stage; (b) an algorithm for the discrimination of broadband noise. The latter is responsible for predictions above $10 \mathrm{MHz}$ in Figure 15, which are flat (except for some isolated peaks discussed below).The reason is that this algorithm removed frequencies where $I_{S 1}$ and $I_{S 2}$ were under the sensitivity of the measurement system (i.e., at noise level). CE sensed by the measurement chain for model validation are actually at noise level above $15 \mathrm{MHz}$, in agreement with predictions. Only in a small frequency range $(10-15 \mathrm{MHz})$ one can observe some measured peaks arising from the noise floor which are not predicted. However, these are very low values of CE, of minor 


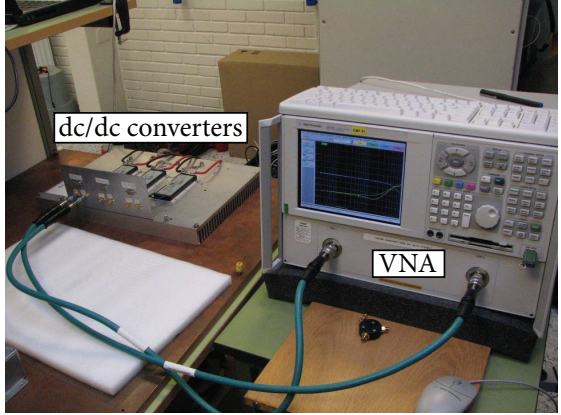

(a)

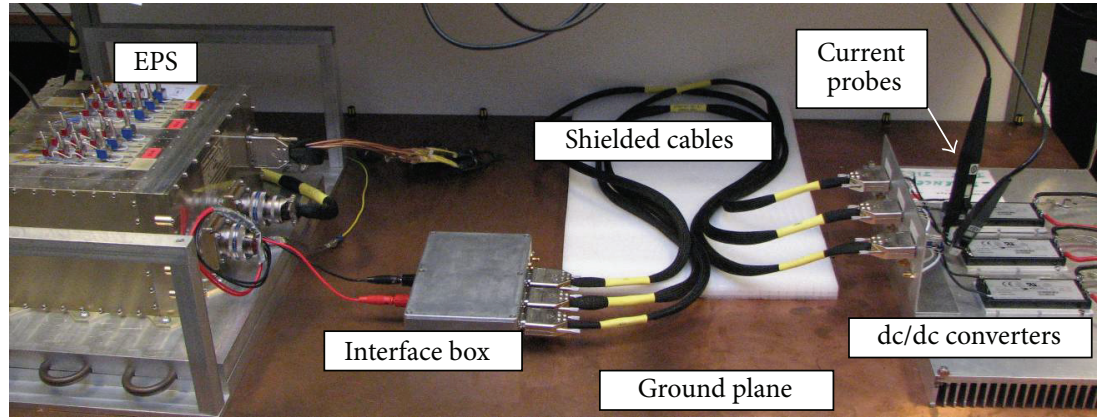

(b)

FIGURE 14: Details of test setups used for validation. (a) dc/dc converters under unit-level testing for the identification of the input admittance in the test setup described in Figure 3(a); (b) the system-level test setup, where dc/dc converters are fed by the PCDU (in the satellite EPS) through shielded TWP cables. CE at cable ends are measured via current probes connected to an oscilloscope.

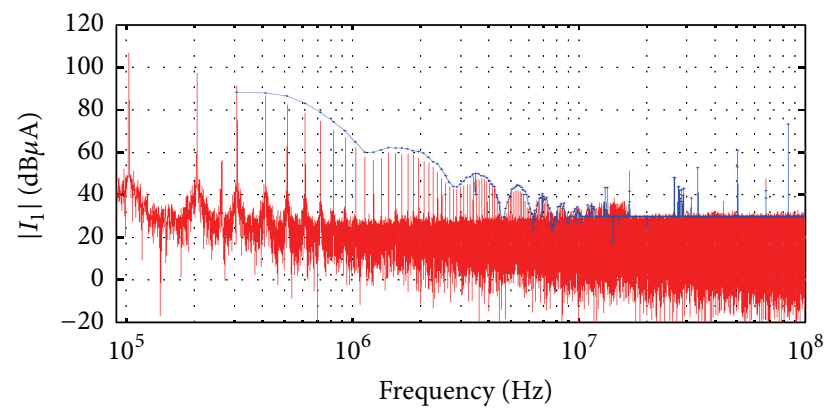

Figure 15: Magnitude of CE (current in the plus dc terminal) of $\mathrm{dc} / \mathrm{dc}$ converter 1 . Red lines refer to experimental measurements (i.e., DFT of currents acquired by the oscilloscope). Blue dots and lines refer to predictions obtained by the proposed model.

relevance as far as EMC purposes are concerned. Finally, concerning isolated peaks appearing in predictions and/or measurements above $10 \mathrm{MHz}$, it was verified that they were not part of the actual spectrum of the $\mathrm{dc} / \mathrm{dc}$ converter CE due to switching activity but originated from external spurious effects (clocks of electronic circuitry, external radiofrequency electromagnetic fields, etc.).

Apart from the discussed spurious effects, predictions and measurements appear to be in good agreement, since the error does not exceed $6 \mathrm{~dB}$ on average.

\section{Conclusion}

EMC is an important technical field in Space engineering, pursuing the prevention of risks associated with electromagnetic emission or susceptibility, which may lead a mission to failure. Generally, EMC is achieved through a balanced combination of good engineering practices, guidelines, simplified analyses and modelling, and a great deal of testing at unit level and throughout all the satellite development phases [1]. Test execution and problems fixing, whenever necessary, imply significant costs so that EMC verification constitutes an expensive and time-consuming element in the frame of satellite qualification activities.
Among major sources of electromagnetic interference in satellites, the operation of $\mathrm{dc} / \mathrm{dc}$ converters is a critical aspect. Requirements on $\mathrm{CE}$ generated by $\mathrm{dc} / \mathrm{dc}$ converters are generally verified at unit level, for example, via dedicated test setups for each unit and subsystem [1]. However, it is well known that the EMC of mission-critical systems composed of several units cannot rely only on a simple combination of unit-level assessments. The interaction of different devices has to be taken into account at system level. Specifically, for $\mathrm{CE}$, there is a need to account for the simultaneous operation of $\mathrm{dc} / \mathrm{dc}$ converters and the impact of the whole satellite power system on the propagation of disturbances, including PCDU and interconnecting cables. If this approach was not followed, there would be a significant risk of underestimating $\mathrm{CE}$ flowing in power buses with possible detrimental effects on signal interconnects which may couple interference through crosstalk or radiated susceptibility.

In line with this aim, a prediction tool for the evaluation of CE in satellite power systems was proposed, which is based on (a) behavioral modeling of dc/dc converters represented as sources of CE in the frequency range $100 \mathrm{kHz}-100 \mathrm{MHz}$; (b) MTL modeling of shielded TWP bundles; and (c) solution of the circuit model of the network of cables and $\mathrm{dc} / \mathrm{dc}$ converters connected to the PCDU, for the computation of currents propagating in the power bus. In particular, the behavioral model of $\mathrm{dc} / \mathrm{dc}$ converters is a linear multiport in the frequency domain, characterized by two current sources and an admittance matrix. Model parameters are identified by two experimental procedures at unit level, with the dc/dc converter tested alone in a controlled test setup. Shielded TWP bundles are modeled by the chain-parameter representation of MTL theory. To this aim, the actual nonuniform MTL with inhomogeneous dielectric medium is simplified through (a) the use of p.u.l. parameters averaged over the spatial twist period of TWPs and (b) the definition of an effective dielectric permittivity. This model proved to be accurate for the reproduction of actual cable parameters measured by a VNA.

Finally, a system-level test setup composed of a PCDU, three shielded cables, and three $\mathrm{dc} / \mathrm{dc}$ converters was modeled and tested to corroborate the validity of the proposed 
modeling approach. Good agreement of predictions and measurements was found, even considering measurement uncertainty related to instrumentation and the uncertainty related to test setup (i.e., geometrical arrangement, connectors, etc.), which has strong impact on voltages/currents at radio frequencies.

For the most significant emission peaks in the frequency spectrum, which are those comparable to limit levels required by EMC specifications [1], the prediction error was lower than $6 \mathrm{~dB}$. This result is in line with the aforementioned technical objective of developing reliable system-level analysis tools that pave the way for the reduction of design iterations and expensive test campaigns in the Space industry, with obvious cost benefits, while safeguarding and possibly increasing reliability and built-in design compatibility. In particular, the proposed simulation tool can provide a valuable and crucial support for (a) the definition of unit-level CE requirements from system-level technical performances; (b) risk reduction and EMC reliability assessment at an early-design stage; and (c) performing system-level trade-off analyses.

\section{Conflict of Interests}

The authors declare that there is no conflict of interests regarding the publication of this paper.

\section{Acknowledgments}

This work was supported by the European Space Agency (ESTEC, Noordwijk, Netherlands) under Contract 21051/07/ NL/GLC. Academic authors wish to thank the aerospace company CGS S.p.A-Compagnia Generale per lo Spazioan OHB company, Milan, Italy, for supporting experimental activities.

\section{References}

[1] ECSS-E-ST-20-07C, "Space Engineering, Electromagnetic Compatibility," European Cooperation for Space Standardization (ECSS), February 2012, http://www.ecss.nl/.

[2] C. R. Paul, Introduction to Electromagnetic Compatibility, John Wiley \& Sons, New York, NY, USA, 1992.

[3] MIL-STD-1541, "Electromagnetic Compatibility Requirements for Space Systems," December 1987.

[4] Department of Defense, "Measurement of electromagnetic interference characteristics," Tech. Rep. MIL-STD-462D, 1993.

[5] V. W. Greb, "Controlling conducted emissions from DC-DC converters," in Proceedings of the EMC ESD International Conference, Denver, Colo, USA, April 1993.

[6] D. L. Johnson, G. T. Smedley, G. R. Mon, R. G. Ross Jr., and P. Narvaez, "Cryocooler electromagnetic compatibility," in Cryocoolers 8, R. J. Ross, Ed., pp. 209-220, Springer, New York, NY, USA, 1995.

[7] F. Torres, A. Reineix, P. Bisognin, and P. Pelissou, "Analyse expérimentale et modélisation théorique des perturbations conduites sur les bus d'alimentation des satellites," in 13ème Colloque International et Exposition sur la Compatibilité Electromagnétique (CEM '06), pp. 310-312, Malo, France, April 2006 (French).
[8] Q. Liu, F. Wang, and D. Boroyevich, "Modular-terminalbehavioral (MTB) model for characterizing switching module conducted EMI generation in converter systems," IEEE Transactions on Power Electronics, vol. 21, no. 6, pp. 1804-1814, 2006.

[9] A. Perez, A. Sanchez, J. Regue, M. Ribo, P. Rodriguez-Cepeda, and F. J. Pajares, "Characterization of power-line filters and electronic equipment for prediction of conducted emissions," IEEE Transactions on Electromagnetic Compatibility, vol. 50, no. 3, pp. 577-585, 2008.

[10] H. M. Rebholz, S. Tenbohlen, and W. Khler, "Time-domain characterization of RF sources for the design of noise suppression filters," IEEE Transactions on Electromagnetic Compatibility, vol. 51, no. 4, pp. 945-952, 2009.

[11] G. Spadacini, D. Bellan, S. A. Pignari, R. Grossi, and F. Marliani, "Prediction of conducted emissions of DC/DC converters for space applications," in Proceedings of the Asia-Pacific Symposium on Electromagnetic Compatibility (APEMC '10), pp. 798-801, Beijing, China, April 2010.

[12] D. Bellan, F. Marliani, S. A. Pignari, and G. Spadacini, "Spectral analysis of conducted emissions of DC/DC converters," in Proceedings of the IEEE International Symposium on Electromagnetic Compatibility (EMC '10), pp. 490-494, Fort Lauderdale, Fla, USA, July 2010.

[13] A.-M. Sánchez, S. A. Pignari, J.-R. Regué, and M. Ribó, "Device modeling for nonstationary conducted emissions based on frequency- and time-domain measurements," IEEE Transactions on Electromagnetic Compatibility, vol. 54, no. 4, pp. 738746, 2012.

[14] G. Spadacini, F. Grassi, F. Marliani, and S. A. Pignari, "Transmission-line model for field-to-wire coupling in bundles of twisted-wire pairs above ground," IEEE Transactions on Electromagnetic Compatibility, vol. 56, no. 6, pp. 1682-1690, 2014.

[15] MIL-STD-462, "Measurement of Electromagnetic Interference Characteristics," July 1967.

[16] C. R. Paul, Analysis of Multiconductor Transmission Lines, John Wiley \& Sons, New York, NY, USA, 1994. 

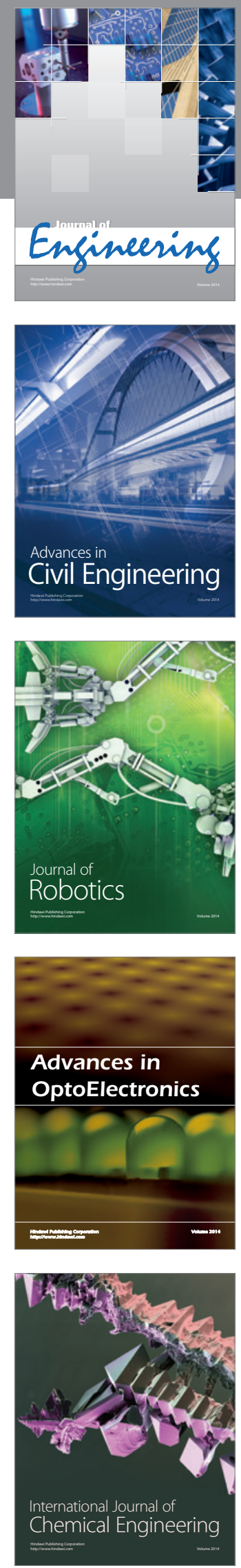

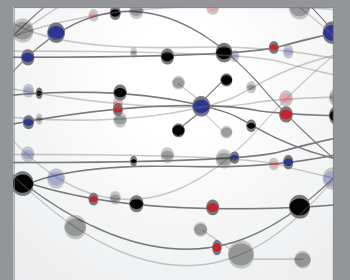

The Scientific World Journal
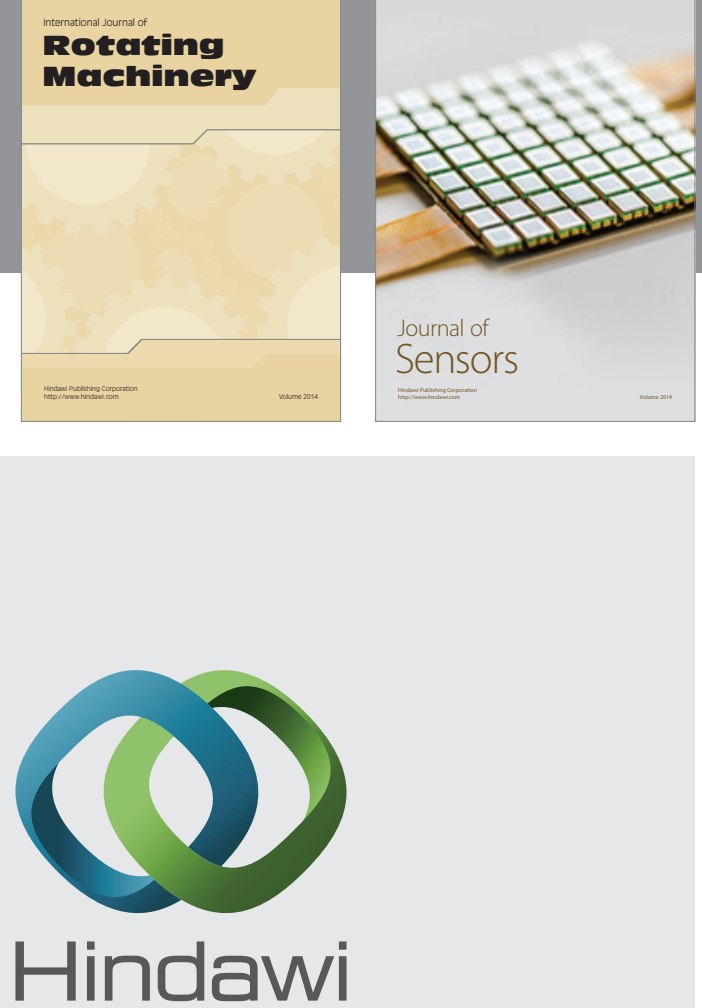

Submit your manuscripts at http://www.hindawi.com
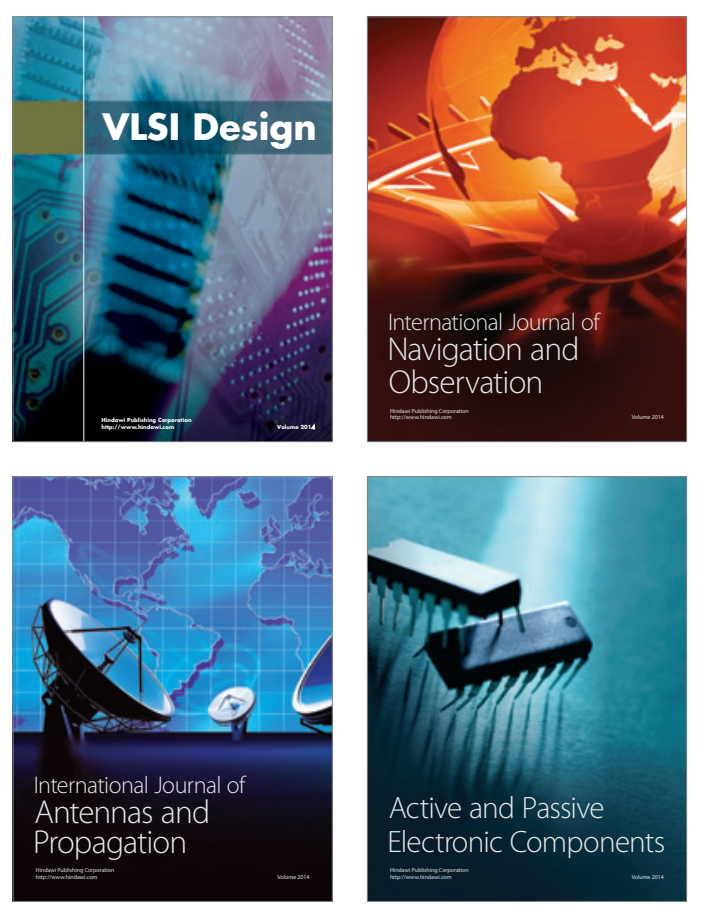
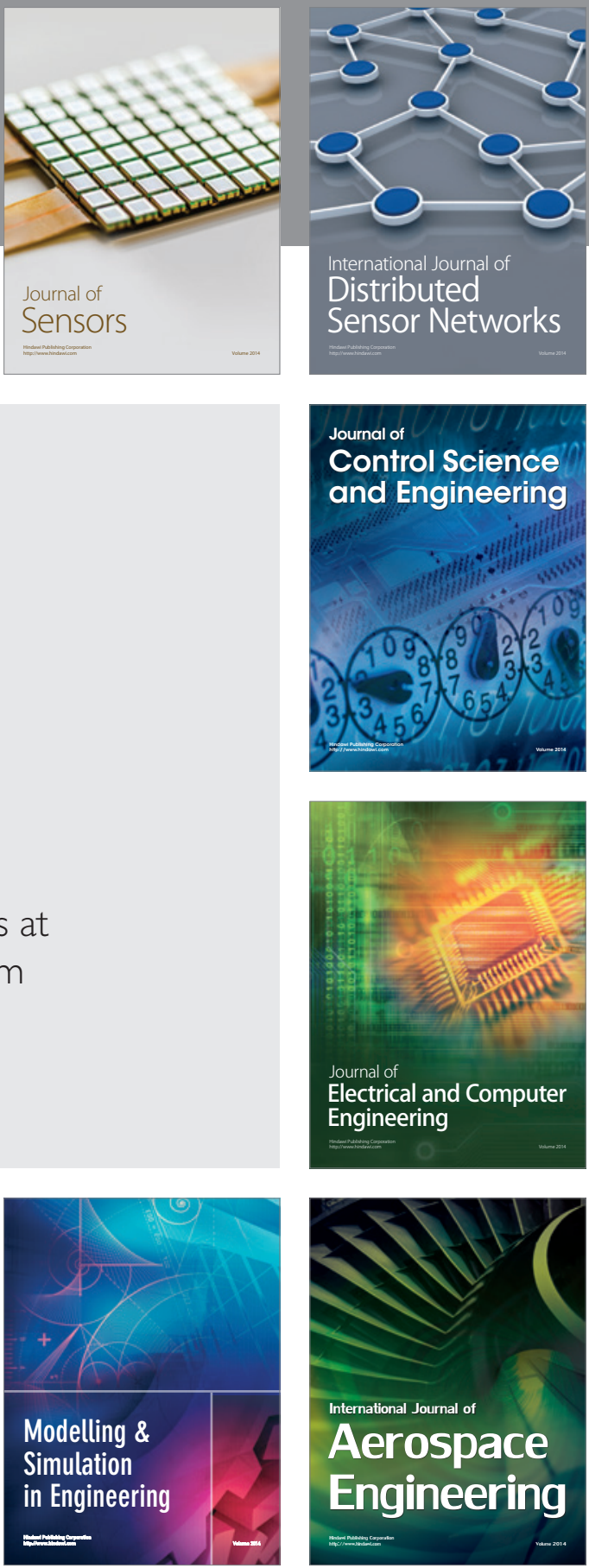

Journal of

Control Science

and Engineering
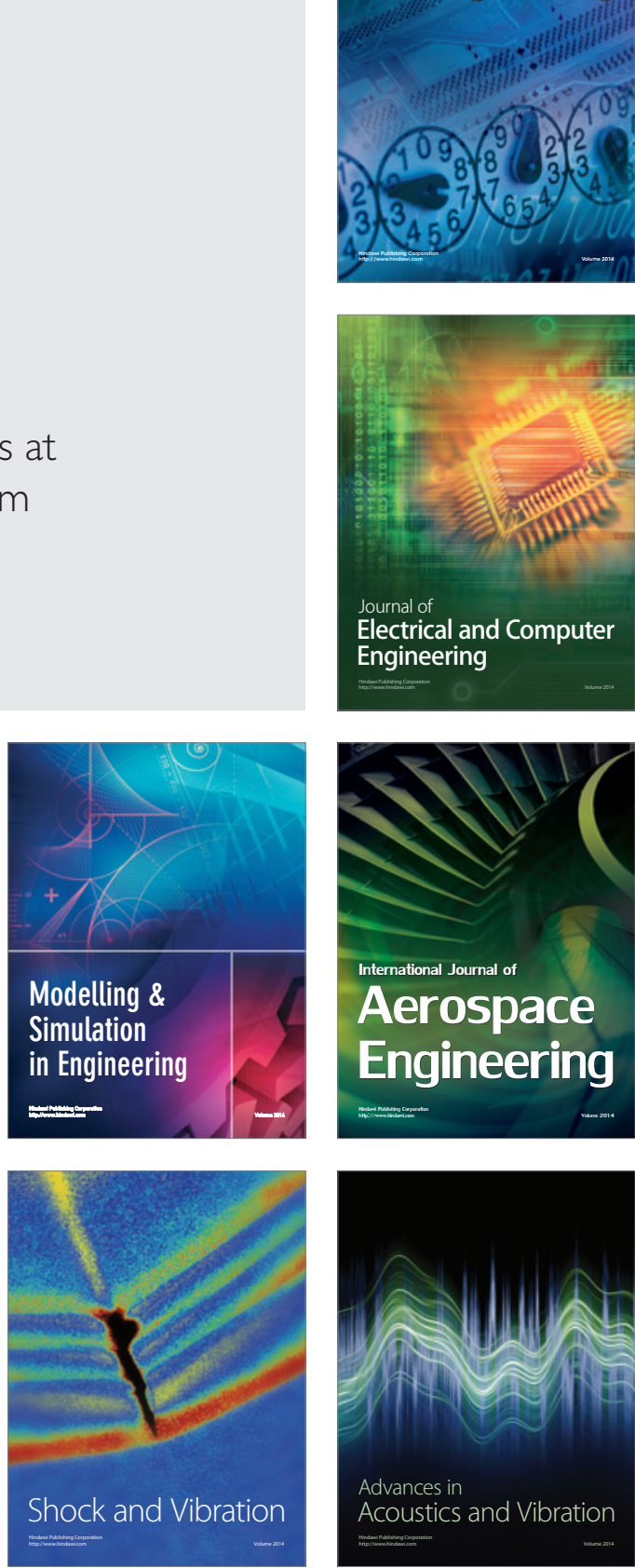